\title{
Segregación espacial en Tarímbaro, municipio periurbano de la zona metropolitana de Morelia, Michoacán, México
}

\section{Spatial segregation in Tarimbaro, municipality in the periurban of Morelia, Michoacán, México}

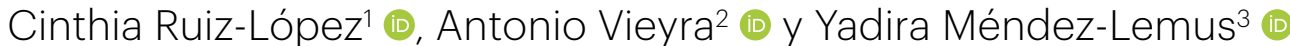

\begin{abstract}
RESUMEN
La segregación espacial cobra importancia por la transición urbana- demográfica en las ciudades. En América Latina y México, los estudios se han centrado en la diferenciación por ingresos y clases sociales; principalmente, en las grandes urbes excluyendo a las de mediano tamaño y su periurbano. La investigación analiza los patrones de segregación espacial en Tarímbaro, municipio periurbano de la Zona Metropolitana de Morelia, a través de los cambios en los atributos personales de la población debido a la expansión urbana y las dinámicas territoriales internas. Se calcularon índices para medir la segregación en cada atributo poblacional, en tres cortes temporales (1990, 2000 y 2010). Concluyendo, la segregación es coproducida por dinámicas propias y externas del territorio, cambiando los patrones de segregación en tres rutas: homogenización del área más antigua del municipio; concentración de población indígena en localidades dispersas; consolidación de una franja homogénea de población recién llegada con condiciones desiguales.
\end{abstract}

Palabras claves: Segregación, ciudades de mediano tamaño, periurbano, población indígena, inmigrante.

\section{ABSTRACT}

Segregation becomes important due to the process of urban and demographic transition. Research about segregation in Latin American region and México has focused on income and social class and those have done in large cities particularity. These studies have overlooked small and middle- sized cities

\begin{abstract}
The paper aims to analyze the spatial segregation pattern in Tarimbaro, using changes in the population's attributes due to urban expansion and internal territorial processes. Segregation was measured through indices for each personal attribute, in three moments $(1990,2000,2010)$. The main conclusion is that segregation is coproduced by internal and external dynamics of the territories, changing the pattern of segregation in three ways: evenness of traditional localities, concentration of indigenous and consolidation the homogeneous area of newly population, everyone's with inequal conditions.
\end{abstract}

Keywords: Segregation, middle-sized cities, periurban, indigenous population, in-migrants population. 
La segregación históricamente ha formado parte de la estructura de las ciudades, expresiones de dicho proceso se pueden encontrar desde la distribución de la población en tiempos coloniales. Es considerada una de las principales líneas de investigación urbana y su estudio se originó en la segunda década del siglo XX, con los aportes de Burgess (1925) en la escuela de Chicago. Recientemente ha renovado el interés de los académicos por procesos como la inequidad social (en ingresos), las políticas públicas, los cambios económicos estructurales y el nivel de conectividad global, considerados como generadores de la segregación a nivel global (Aguilar y Lopez, 2018; Hill y Brennan, 2005). Estos procesos reestructuran a las ciudades y su orden espacial, cambiando los índices y formas de segregación (Savage et al., 2013; Sabatini, 2015), volviendo a las ciudades altamente fragmentadas (Bähr y Borsdorf, 2005; Janoschka, 2002).

Se entiende a la segregación como un proceso de diferenciación de una población por sus características sociales (pertenencia a un grupo étnico, ingreso, capacidad de ahorro y las condiciones de la vivienda). Tiene dos dimensiones de representación, una espacial y otra social (García y Rodríguez, 2017; Vergara- Erices y Garín, 2016). La primera perspectiva, la espacial, se relacionada con la distribución de la población en el territorio y se centra en las diversas formas en que una población se distribuye. Algunos autores plantean que existen al menos cinco dimensiones que expresan la segregación que son: igualdad, exposición, concentración, centralidad y agrupamiento (Massey y Denton, 1988; Massey, Rothwell y Domina, 2009; Domínguez, 2017). Para identificar las dimensiones en que una población está segregada se han creado, desde hace más de cuarenta décadas y guiados por la literatura anglosajona índices que permiten expresar la disimilitud en la distribución poblacional, los cuales serán abordados más adelante.

La otra perspectiva es la social, centrada en la diferencia en las interacciones sociales, políticas, culturales, ambientales entre grupos sociales, caracterizada por su identidad, prestigio y percepción del territorio (Sabatini, 2006; Savage et al., 2013), perspectiva que no se desarrollará en esta investigación.

La segregación hace referencia a la representación de un grupo, respecto al total de la población en un territorio, en un tiempo determinado y con implicaciones sociales en las comunidades, como el acceso a servicios y oportunidades para la población. Es decir, el acomodo de la población en el territorio tiene una expresión en la estructura espacial urbana (Sabatini 2006; Madrazo y Kempen, 2011; Wu et al., 2014; Aguilar, Romero y Hernández 2015).

Históricamente, el abordaje de la segregación, principalmente espacial, ha estado relacionado con la ubicación geográfica, por ejemplo, en Europa (Bolt y Kempen, 1997) y Estados Unidos (Massey y Denton, 1988; Massey, Rothwell y Domina, 2009) se ha estudiado la diferenciación, principalmente, desde las características étnicas de los individuos. De diferente manera, en los países de América Latina (Aguilar, Romero y Hernández, 2015; Sabatini, 2015; Schteingart, 2015), África (Badawy, Abdel-Salam, Ayad, 2015) y Asia (Madrazo y Kempen, 2011; Wu et al., 2014) se han centrado principalmente en la diferenciación por ingresos y clases sociales, siendo estos muy escasos (Wu et al., 2014; Madrazo y Kempen, 2011) y poco abordados desde las diferencias étnicas, de migración y etarias.

Los países en desarrollo, principalmente localizados en Asia, África y América Latina, se caracterizan por su pujante crecimiento urbano expresado en la concentración poblacional en las 
grandes aglomeraciones urbanas impidiendo un crecimiento equilibrado, particularmente en sus periferias (UCLG y CGLU, 2016). La población de estas zonas, de transición urbano-rural, se enfrentan a diversos retos por la migración, desempleo y carencia en servicios (Smith, 2018). En particular, en la región de América Latina, una de las más urbanizadas a nivel mundial con el $80 \%$ de la población viviendo en áreas urbanas (UN, 2014), se reconocen dos procesos importantes en el crecimiento urbano: las dinámicas de crecimiento nacionales y la migración urbano-rural (Da Gama, 2011). Esta situación modifica la distribución de la población, principalmente en el territorio que rodea a la ciudad, donde cambian los patrones de segregación espacial.

El espacio que rodea a la ciudad, denominado periurbano, es coproducido por diversos procesos y divisiones sociales expresadas espacialmente por la segregación. Dicho espacio, se vuelve relevante al ser una interfaz urbano-rural, caracterizado por múltiples conexiones funcionales entre lo urbano y lo rural (económicas, de mercado, de viaje, de trabajo, de capacitación, de servicios, de bienes, de materiales, de capital, entre otras) y medios de vida (Parr, 2005; Nelles, 2012). El periurbano no se puede delimitar por sus fronteras político-administrativas, ya que incorpora gradualmente a sus dinámicas las transformaciones producto de la cercanía de la ciudad. Por lo tanto, el periurbano está compuesto de estructuras tradicionales (Nello, 1998), espaciales y sociales modificadas por la integración a la ciudad, generando nuevas formas urbanas y, por lo tanto, nuevas formas de segregación (Lichter y Brown, 2011). Particularmente, el periurbano latinoamericano es heterogéneo, compartido por distintos tipos de población con vivienda de clase alta, media alta y baja o precaria; una diversidad de funciones con espacios productivos y de consumo conectados a la ciudad por medio de ejes viales (Sabatini, 2006).

Específicamente, en ciudades de tamaño mediano ${ }^{4}$, la estructura urbana es dominada por una ciudad central rodeada por un cinturón periurbano formado por pueblos antiguos, villas, áreas agrícolas y forestales, entre otros; que, a diferencia de las grandes urbes, mantienen características fuertemente rurales en la población, en los paisajes y en las viviendas (Cavailhés, Peeters, Sékeris y Thisse, 2004). El incremento de la población de los municipios periféricos añade demandas adicionales para la infraestructura urbana y las viviendas, el acceso a servicios y empleo, y la seguridad de tenencia de la tierra. Por lo que estos espacios muchas veces presentan carencias en infraestructura y servicios, y son lugares emisores de trabajadores con baja educación y capacitación (Hill y Brennan, 2005). En el contexto mexicano, el periurbano, es habitado por población que trabaja en gran proporción en la ciudad central (Álvarez de la Torre, 2017; Domínguez, 2017).

Además, el periurbano experimenta modificaciones en las estructuras tradicionales espaciales y sociales; su población vive las nuevas divisiones sociales; su crecimiento con bajas densidades y alta desocupación habitacional incrementa los costos en infraestructura y lejanía con los mercados laborales. Estos procesos convierten al periurbano en un territorio socialmente heterogéneo que modifica los patrones de segregación.

Según Badawy et al. (2015) y Sabatini (2015) la reconfiguración de la estructura en ciudades latinoamericanas ha cambiado el espacio usado por la población, con la descentralización de las

El criterio para clasificar a las ciudades de mediano tamaño cambia en el país a país. Algunos consideran que estas urbes tienen más de 500 mil habitantes (Roberts, 2015); para otros, esta categoría oscila de 50,000 a 1 millón de habitantes, específicamente en América Latina y el Caribe las ciudades de mediano tamaño están principalmente en el rango de 300 mil a 1 millón (UCLG y CGLU, 2016). 
elites en urbanizaciones cerradas en las periferias, lugar ocupado históricamente por población pobre y barrios informales. Incrementando la proximidad física entre grupos sociales junto a sus diferencias social (Sabatini y Brain, 2008).

En México, al igual que en la región Latinoamericana, desde los noventa presenta el mayor dinamismo económico en las ciudades de tamaño mediano ${ }^{5}$ y un rápido crecimiento de población en el periurbano, principalmente por familias pobres, migrantes (Da Gama, 2011; Roberts, 2015), e indígenas. Este país se distingue en la región latinoamericana, porque junto con otros países latinoamericanos como Chile, presenta alta urbanización, donde el periurbano sigue creciendo con menos informalidad y experimentando cambios en su organización urbana (Da Gama, 2011).

Las ciudades de tamaño mediano mexicanas son disímiles, pero presentan temas constantes como la participación en la economía nacional; precarización en las formas de vida y dispersión en el espacio periurbano (Roberts, 2015; Álvarez de la Torre, 2017; Aguilar y López, 2018). Esta situación modifica la distribución espacial de la población que tendrá implicaciones en la segregación e interacción entre los grupos sociales (Álvarez de la Torre, 2017; Domínguez, 2017). De acuerdo con ello, el periurbano de ciudades de tamaño mediano mexicanas actualmente experimenta la llegada de población de bajos ingresos y con la permanencia de habitantes tradicionalmente rurales, que coproduce nuevas formas de segregación.

Los estudios sobre la segregación en México se han centrado en las grandes ciudades como la Ciudad de México (Aguilar y Mateo, 2011; Schteingart, 2015), Guadalajara (Schteingart, 2015); Monterrey (Schteingart, 2015; Aguilar, 2004) y Puebla (Hernández et al., 2009) que han usado algunas características socioeconómicas de los habitantes, que no necesariamente dan cuenta de los complejos cambios que experimentan los atributos personales de la población y las formas en que se reproduce la segregación en los municipios periféricos, o el espacio que construye el periurbano.

Por otro lado, las escasas investigaciones de segregación en las ciudades de tamaño mediano mexicanas han abordado el análisis desde las clases sociales, la distribución de población por edades y la vivienda en renta (Domínguez, 2017; Álvarez de la Torre, 2017). Estas investigaciones si bien han contribuido al conocimiento de la segregación en México, no han analizado ni discutido las modificaciones que ocurren en el espacio periurbano por la superposición de nuevas dinámicas urbanas y antiguas estructuras socio-espaciales y sus implicaciones a nivel municipal. La importancia de estudiar las urbes de tamaño mediano es que replican la complejidad de las grandes ciudades, intensificando la fragmentación y segregación en espacios periurbanos.

En esta investigación las principales interrogantes fueron cómo se reconfigura el patrón de segregación en un municipio periurbano de la Zona Metropolitana de Morelia por los cambios en los atributos personales de la población, producto de la expansión urbana; y sus implicaciones en el acceso a servicios y condiciones de la vivienda.

En México, las ciudades se clasifican en centros urbanos, conurbaciones urbanas y zonas metropolitanas. En este contexto, las ciudades de tamaño mediano son de dos tipos: de 100 mil a menos de 500 mil habitantes (medias); y de 500 mil hasta 1 millón de habitantes (intermedias), estas últimas son todas Zonas Metropolitanas (SEDESOL, CONAPO, INEGI 2010 citado en SEDESOL, ONU-HABITAT, 2011). 
Por ello, el objetivo principal fue analizar los cambios en el patrón de segregación en un municipio periurbano en la Zona Metropolitana de Morelia (ZMMor), ciudad media, a partir de la disimilitud en los atributos personales y sus implicaciones en el acceso a servicios y condiciones de las viviendas.

El argumento principal que guía a este estudio es que los cambios en los atributos personales de los habitantes del periurbano son generados por la expansión urbana y dinámicas propias de la estructura social tradicional, reajustando los patrones en tres direcciones: la homogenización de la parte más antigua del municipio, la concentración de la población indígena en localidades dispersas y la creación de una franja de nuevas tipologías de segregación más desiguales.

Este artículo pretende evidenciar la heterogeneidad de los atributos personales de la población, de un municipio periurbano de la ZMMor, ciudad de tamaño mediano; y cómo ello, guía las rutas que toman los patrones de segregación. Si bien, se reconoce que la estructura social de la población es determinante para la segregación, este artículo pretende mostrar cómo el proceso de periurbanización agudiza las desigualdades territoriales previamente construidas. Lo anterior es de importancia para la gestión de la urbanización en municipios periurbanos donde los gobiernos locales afrontan dificultades para el ordenamiento del territorio.

La propuesta metodológica visualiza las diferentes dimensiones y temporalidades de la segregación en el periurbano, usando los atributos personales, como una categoría de análisis, que supera las limitaciones de medir la segregación únicamente por el ingreso o clase, y permite visibilizar mejor las diversas expresiones de la segregación entre los grupos sociales (por rasgos socioeconómicos, étnicos y de migración), así como las formas (segregación, exposición, concentración y centralidad) cambiantes con el tiempo, a partir de tres cortes temporales (1990, 2000 y 2010), retomados de los Censos de Población y Vivienda del Instituto Nacional de Estadística y Geografía (INEGI).

\section{La segregación en el periurbano norte de la ZMMor: Caso de municipio de Tarímbaro}

En México, el crecimiento urbano en las ciudades de tamaño mediano es diferencial. Morelia es una de las urbes que ha mantenido su crecimiento de 1990 al 2010; se localiza en una de las regiones con mayor crecimiento del Producto Interno Bruto (PIB) del país. Generando una expansión sobre territorio rural, que según Martínez y Monroy-Ortiz (2009: 38) de 1995 al 2005 correspondió a la ocupación de tierra productiva de 800 a 1,700 km², rebasando los límites municipales y trastocando a otros municipios como Tarímbaro y Charo; los cuales forman parte de la Zona Metropolitana de Morelia (ZMMor). Tarímbaro se localiza al Norte de Morelia, su ubicación es estratégica al ser paso obligado de Morelia a otras entidades como Guanajuato (carretera 43), San Luis Potosí (carretera 120) y la vía a Guadalajara (la tercera urbe más importante en el país).

La relación entre Tarímbaro y Morelia es añeja, en la época colonial el primero suministraba mano de obra, productos agrícolas y ganaderos importantes para la especialización rural de Morelia. El municipio estaba organizado en haciendas y ranchos $^{6}$ que se mantuvieron casi intactos 
aún en periodos de crecimiento de Morelia como el ocurrido de 1940 a 1970 (Vargas, 2014). Fue en 1980 cuando Tarímbaro experimentó un crecimiento desordenado producto de la expansión hacia el norte de Morelia por la llegada de población de Ciudad de México, Guadalajara y Querétaro; el segundo periodo de crecimiento en Tarímbaro ocurrió de 1990 al 2000 con la creación de nuevos fraccionamientos; por último, del 2000 al 2010, Tarímbaro creció 6.9\% (INEGI, 2000, 2010) la tasa más alta de la ZMMor. Históricamente este crecimiento ha sido en la colindancia con Morelia, de 1990 al 2010 las localidades que más crecieron y se crearon en ese periodo están marcadas con círculos en la figura 1.

Figura No 1.

Crecimiento histórico de las localidades de Tarímbaro

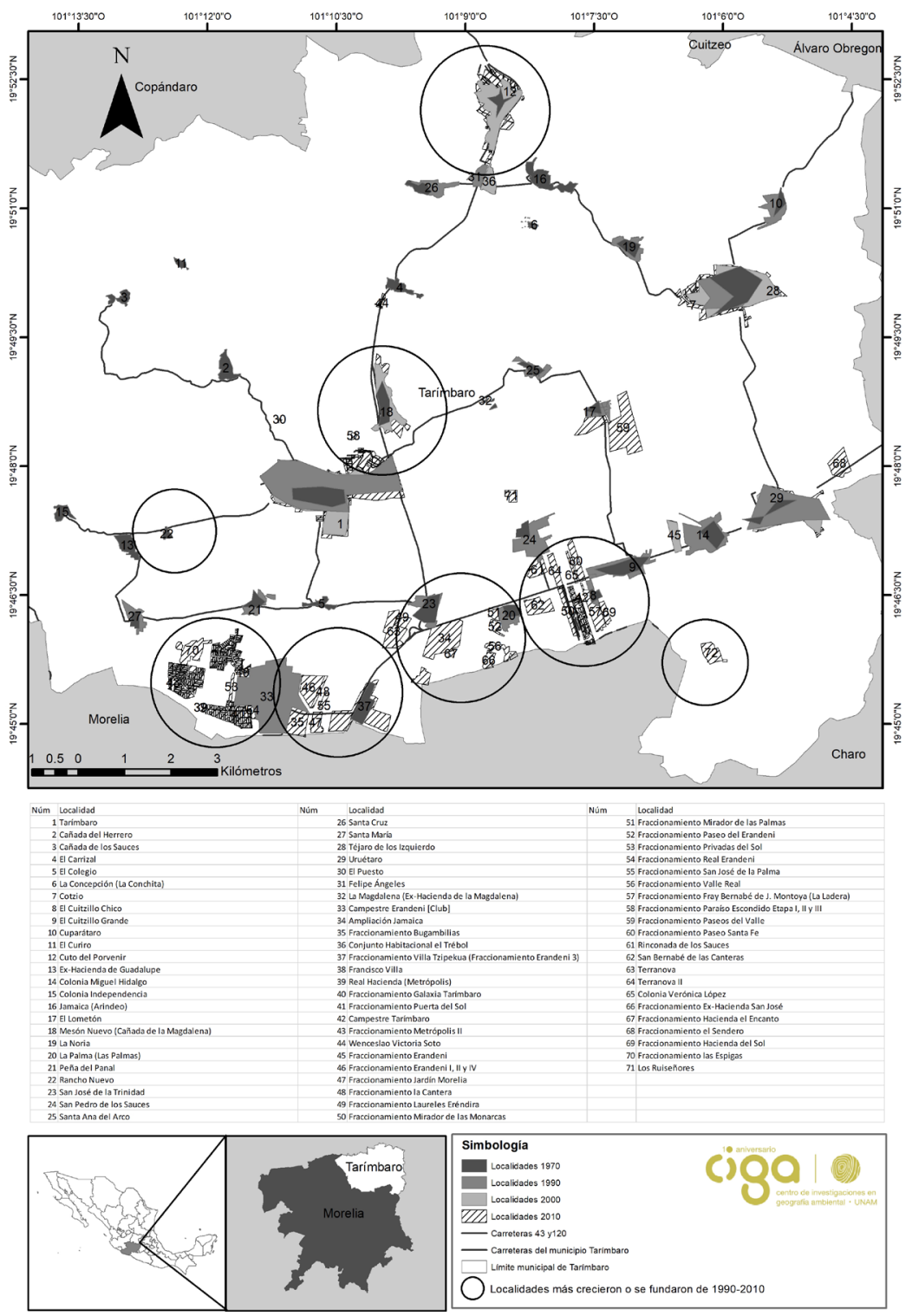

Fuente: Elaboración propia con base a INEGI (1990, 2000, 2010). 
Pero el rápido crecimiento poblacional no ha estado acompañado de infraestructura, equipamiento y vías de comunicación que mejoren la calidad de vida de la población. Del 2005 al 2010 el mayor crecimiento poblacional fue en localidades sin drenaje, además, esas poblaciones carecen de equipamiento escolar que ha tardado hasta cuatro años para edificarse. Estos datos muestran que la expansión urbana desordenada reproduce desigualdades en el periurbano, principalmente en la inmediación con Morelia, ocasionando modificaciones en las formas de segregación espacial que viven los pobladores recién llegados y originarios de Tarímbaro.

\section{Metodología para analizar la segregación desde los atributos personales de la población del periurbano}

Esta investigación considera a la segregación como un proceso que cambia en una temporalidad determinada (Massey, Rothwell y Domina, 2009) y se expresa a diferentes escalas y dimensiones en el periurbano. Por ello, para analizar la segregación se usaron los atributos personales de los habitantes del municipio de Tarímbaro que más han cambiado de 1990 al 2010. Ello pretende superar las limitaciones de otros estudios que a partir del análisis factorial reducen las variables a: ingresos, nivel educativo, ocupación y vivienda (Rubalcaba y Schteingart, 2012; Savage et al., 2013; Aguilar, Romero y Hernández, 2015). La metodología se realizó a través de los siguientes pasos: Primero, se identificaron los atributos personales de la población del municipio de Tarímbaro, divididos en socioeconómicos, de migración y étnicos (Cuadro No 1).

Cuadro No 1.

Atributos personales de los habitantes de Tarímbaro

\begin{tabular}{|l|l|c|c|c|}
\hline $\begin{array}{l}\text { Atributos } \\
\text { personales }\end{array}$ & \multicolumn{1}{|c|}{ Estratificación } & \multicolumn{3}{c|}{$\begin{array}{c}\text { Población en el } \\
\text { municipio (\%) }\end{array}$} \\
\hline & & $\mathbf{1 9 9 0}$ & $\mathbf{2 0 0 0}$ & $\mathbf{2 0 1 0}$ \\
\hline \multirow{5}{*}{ Socioeconómicos } & Población de 15 años y más con escolaridad básica & 10.80 & 3.07 & 13.76 \\
\cline { 2 - 5 } & Población de 15 años y más sin escolaridad básica & 10.55 & 43.59 & 3.57 \\
\cline { 2 - 5 } & Población joven (0-14 años) & NA7 & 34.69 & 29.39 \\
\cline { 2 - 5 } & Población adulta (15 a 59 años) & NA & 27.24 & 63.96 \\
\cline { 2 - 5 } & Población de 50 años y más & NA & 38.07 & 6.1 \\
\cline { 2 - 5 } & Población sin servicio de salud & NA & 82.08 & 33.8 \\
\cline { 2 - 5 } & Población económicamente activa ${ }^{8}$ & 24.61 & 25.40 & 35.67 \\
\cline { 2 - 5 } & Población desocupada & 39.94 & 43.49 & 1.95 \\
\hline Migración & Población nacida en otro estado & NA & 4.13 & 10.89 \\
\hline \multirow{2}{*}{ Étnico } & Población de 5 años y más que en el anterior cen- & NA & 85.27 & 76.73 \\
\hline & so vivía en otro estado & & & \\
\hline & Población que habla alguna lengua indígena & 0.12 & 0.18 & 0.32 \\
\hline
\end{tabular}

Fuente: Elaboración propia con base en INEGI (1990, 2000, 2010).

En el censo de 1990 no existen datos de población dividida por rangos de edades. Los grupos etarios se formaron por la disponibilidad de datos en los Censos. En el 2000, los rangos fueron de 0 a 14 años, de 15 a 49 años y más de 50 años; mientras en el 2010 los rangos etarios eran de 0 a 14 años, de 15 a 59 años y más de 60 años. De acuerdo con ello, los resultados expresarán los cambios en la segregación por edades.

8 El INEGI, define a la población económicamente activa como aquellos habitantes de 15 años hasta 64 años que dependen de un empleador y la población desocupada quienes no buscan trabajo y no tienen vínculos con empleador (INEGI, 2018). 
Segundo, se definieron las unidades de análisis, las cuales fueron localidades y fraccionamientos $^{9}$, que han tenido una construcción histórica en común y por sus comportamientos similares permiten determinar los patrones de segregación municipal.

Tercero, bajo el argumento que la población puede estar segregada desde diferentes dimensiones: igualdad o uniformidad; exposición o aislamiento; concentración; centralización; y el agrupamiento (Massey y Denton, 1988; Massey, Rothwell y Domina, 2009; Domínguez, 2017). Se usaron las siguientes dimensiones para medir la segregación:

- La igualdad se entiende como la distribución equitativa de una población en todas las localidades del municipio respecto a los grupos de población. Medidas a través de dos índices: de segregación (IS) para determinar la segregación o uniformidad en la distribución de población minoritaria y mayoritaria en las unidades geográficas en que se divide un territorio; y el índice de disimilitud (ID) para comparar la proporción de la población mayor con respecto al grupo minoritario (Ducan, Ducan 1955 citado en Massey y Denton, 1988; Massey, Rothwell y Domina, 2009; Domínguez, 2017). La interpretación es el porcentaje de individuos que deben cambiar su ubicación para una distribución uniforme.

La exposición mide el contacto potencial o la posibilidad de interacción entre dos grupos. Se usó el índice de aislamiento (xPx) referente a la probabilidad de que un individuo del grupo ( $\mathrm{x}$ ) comparta la unidad espacial (localidad/fraccionamiento) con otro del mismo grupo $(\mathrm{x})$; $y$ el de interacción ( $\mathrm{xPy}$ ), referente a la probabilidad de que un miembro del grupo (x) se exponga a otro (y) (Massey y Denton, 1988; Massey, Rothwell y Domina, 2009; Domínguez, 2017).

- La concentración se refiere a la cantidad física de espacio urbano ocupado por un grupo. Se usó el índice Delta (Massey y Denton, 1988; Massey, Rothwell y Domina, 2009; Domínguez, 2017) interpretado como el porcentaje de la población que debe cambiar su residencia para tener una densidad uniforme; a mayor concentración la segregación aumentará.

La centralidad es la proporción de la población que reside en el centro respecto de la total (Massey y Denton, 1988). En este caso, se eligió usar a la cabecera de Tarímbaro como el centro de referencia.

De cada atributo personal de la población de las localidades del municipio de Tarímbaro se calcularon los IS, ID, xPx, xPy, Delta, y Centralidad, en los años 1990, 2000 y 2010. El rango utilizado para cada índice fue de 0 al 1; los parámetros para interpretar los índices y el grado de segregación fueron: baja, menor a 0.3; media, de 0.3 a 0.6; y alta mayor a 0.6 (Aguilar y Mateos, 2011; Domínguez, 2017). Ello permitió identificar como la segregación, a partir de los índices, ha disminuido o aumentado, así como, reconocer qué población experimentó esos cambios y dónde se localiza en el territorio del municipio de Tarímbaro.

Esta decisión está sustentada en la disponibilidad de la información en los Censos de población de las publicaciones del INEGI y otros organismos. 
Para representar la distribución espacial de los atributos personales de la población se usó el coeficiente de localización (Sargent Florence citado en Domínguez, 2017) que representa la proporción de un grupo respecto al total en la localidad y en el municipio.

La estandarización de los atributos personales se realizó a través de la puntuación " $Z$ " o Z-score, permitiendo comparar puntuaciones de sujetos con diferente denominador (Aguilar y Mateos, 2011), a partir de obtener el número de desviación estándar con respecto a la media. De acuerdo con ello, los valores "cero" significan el mismo valor que la media municipal; los valores negativos y cercanos a -1 indican una subrepresentación, es decir, la ausencia del atributo; mientras los valores mayores a "uno" significan sobre-representación, el rasgo es mayor en la unidad respecto a la media municipal.

Por último, se eligieron los atributos que experimentaron más cambios en el índice de segregación (IS); y se formaron los grupos que tenían comportamientos similares, usando la herramienta $k$-means, del programa estadístico SPSS. Los grupos mostraron características de las viviendas y los rasgos socioeconómicos de la población, así como, los cambios en los patrones de segregación evidenciando desigualdades que experimentan esos pobladores.

\section{Patrones y cambios de la segregación en Tarímbaro}

En el municipio de Tarímbaro se experimenta una disminución de los índices de segregación de 1990 al 2010, determinado por dos razones: la llegada de población de Morelia, especialmente considerada pobre según sus ingresos ${ }^{10}$; así como, los ajustes en la estructura social de las comunidades tradicionales. Las disminuciones más significativas ocurrieron en población que hablaba una lengua indígena, con escolaridad básica y económicamente activa; mientras los índices aumentaron en la población desocupada, nacida en otra entidad y mayor de 50 años.

\section{Población indígena}

El índice de segregación que más disminuyó durante los 20 años fue la población de 5 años y más que hablaba una lengua indígena, la cual pasó de 0.84 a 0.70 , manteniendo valores altos y una distribución segregada de estos habitantes en el territorio. Aunado a ello, esta población experimenta aislamiento, debido a que su distribución ha pasado de media a altamente concentrada; y alejada del centro del municipio.

En 1990 esta población indígena tenía una distribución más igualitaria (0 a 2 desviación estándar) y solo la localidad La Concepción (\#6) tenía la mayor sobrerrepresentación (4.31). En el 2000, el Norte y Este del municipio concentraban la mayor cantidad de población (Tarímbaro (\#1), Cañada del Herrero (\#3), Cuto del Porvenir (\#12), Colonia Miguel Hidalgo (\#14), Téjaro de los Izquierdos (\#28), El Puesto (\#30) y Uruétaro (\#29)) de 0 a 2.74 desviaciones estándar; teniendo la mayor sobrerepresentación en asentamientos cercanas a vías de comunicación principales

En México, el salario mínimo es de 4 dólares por día, es decir, 120 mensuales. Una población considerada en pobreza por ingresos es aquella que gana menos de 564 dólares mensuales. En el 2014, se estimó que el 53.2\% de la población estaba debajo de la línea de la pobreza (CONEVAL, 2017). 
(El Cuitzillo Grande (\#9)). En el 2010, la población se incrementó dos veces concentrándose en nuevos asentamientos (Fraccionamiento: Erandeni (\#45), Hacienda del Sol (\#69) y Paseo Santa Fe (\#60)) y en localidades originales (Colonia Miguel Hidalgo (\#14) y Santa Ana del Arco (\#25)) con sobrerepresentación respecto a la media municipal (hasta 0.87 desviación estándar).

Resalta el caso de la localidad El Puesto (\#30) que tenía la representación más alta en el municipio (8 desviación estándar). La alta concentración de población indígena aunado a la carencia en servicios de salud y población desocupada, incrementan la desigualdad de la población indígena respecto a otras zonas del municipio que tiene servicios y oportunidades laborales. Esto muestra que el incremento de la población indígena en el 2010 tuvo una distribución disímil, concentrada en una localidad, evidenciando como este grupo tiene una localización menor en nuevos fraccionamientos y en localidades del Norte del municipio.

\section{Población con escolaridad básica}

El índice de segregación de la población de 15 años y más con escolaridad básica se redujo de 0.56 en 1990 a 0.51 en el 2010, permaneciendo en valores medios y mostrando una distribución disímil de esta población en el territorio.

La disimilitud de estos habitantes, con respecto a los pobladores sin educación básica mejoró, en 1990 el $30 \%$ de los habitantes debían cambiar su residencia para una distribución más equitativa; y en el 2010 esta cifra se redujo a 13\%. Al mismo tiempo, estos pobladores tienen mayor posibilidad de contacto con miembros del mismo grupo y habitantes sin escolaridad, debido al bajo aislamiento (0.15). De acuerdo con el último censo de 2010, estos habitantes se han distribuido de manera concentrada pero alejados del centro municipal.

En 1990, la población con escolaridad básica se ubicaba principalmente en localidades tradicionales con valores arriba de la media (con 1 y hasta 2 desviación estándar), como Uruétaro (\#29), El Cuitzillo Chico (\#8) y El Cuitzillo Grande (\#9). En el 2000 el rango de concentración fue de 0 a 2.96, las localidades con mayor concentración estaban cercanas a vías principales como El CuitziIlo Grande (\#9) y Felipe Ángeles (\#31). Mientras en el 2010, la ubicación fue más concentrada con valores de 0 a 2.68 desviación estándar, en localidades contiguas a la carretera, principalmente nuevos fraccionamientos (Hacienda El Encanto \#67, Fraccionamiento Metrópolis II \#43) y localidades tradicionales (El Cuitzillo Chico y El Cuitzillo Grande), la localidad con mayor representación fue Wenceslao Victoria Soto (\#44), cerca de las principales vías de comunicación.

\section{Población económicamente activa y desocupada}

La población ha presentado una segregación media en el territorio con respecto a su ocupación económica, manteniéndose de 1990 al 2010 en la económicamente activa (0.5) e incrementándose en la desocupada, la cual pasó de 0.50 a 0.56.

La disimilitud entre ambos grupos se incrementó en 1990 el 7\% de la población debía cambiar su residencia para una distribución igualitaria, mientras en el 2010 fue del $25 \%$.

El aislamiento que experimentaban esos grupos se invirtió, en 1990 la población desocupada tenía más posibilidades de contacto con habitantes del mismo grupo, pero en el 2010 la po- 
blación económicamente activa incrementó sus posibilidades de interactuar con pobladores del mismo grupo y la población desocupada.

Los cambios en el aislamiento se relacionan con el aumento de la concentración que experimentaron ambas poblaciones; así como, la baja ocupación del centro del municipio. En 1990, la población económicamente activa se distribuía en el territorio de dos formas: menor a la media municipal (menos de 0 desviación estándar) en localidades tradicionales (Cuto del Porvenir (\#12) y El Cuitzillo Chico (\#8)); y hasta dos veces la media municipal (hasta 1.86 desviaciones estándar) en localidades tradicionales más alejadas de las principales vías de comunicación (El Carrizal (\#4), Rancho Nuevo (\#22) y San José Trinidad (\#23)).

En el 2000 los valores de representación de hasta 1 desviación estándar, fueron en localidades tradicionales (Ex hacienda Guadalupe (\#13) y El Cuitzillo Grande (\#9)); y la mayor representación fue en Campestre Erandeni (\#33) con 2.39 desviación estándar, población que se distinguía por tener ingresos económicos que superaban dos o tres veces al resto del municipio. Esto evidenciaba una concentración media de población económicamente activa y con altos ingresos (6 a 10 Salarios Mínimos Mensuales).

En 2010 la representación de la población económicamente activa fue principalmente en fraccionamientos contiguos a la carretera y cerca de la frontera con el municipio de Morelia (Ex Hacienda San José (\#66), Paraíso Escondido (\#58), Laureles Eréndira (\#49) y Hacienda El Encanto (\#67)) y en baja proporción en localidades tradicionales (Francisco Villa (\#38), Téjaro de los Izquierdos (\#28) y Colonia Miguel Hidalgo (\#14)).

Contrario a ello, la población desocupada se ha distribuido alejada de la frontera con Morelia. En 1990 se ubicaba en localidades tradicionales, en dos rangos de representación: menor a la media municipal (menos 0 desviación estándar) en pequeñas localidades tradicionales alejadas del centro (Cañada del Herrero (\#2), Cañada de los Sauces (\#3) y Colonia Independencia (\#15)) y por encima de la media municipal (de 1 a 2 desviación estándar) en localidades alejadas del centro del municipio (La Concepción (\#6), Cuparátaro (\#10), Colonia Miguel Hidalgo (\#14), Jamaica (\#16) y El Lometón (\#17), Santa Ana del Arco (\#25)).

En el 2000, la situación era muy similar, la cabecera de Tarímbaro (\#1) tenía una representación menor a la media municipal (menos de 0 desviación estándar), la más alta representación fue en localidades alejadas de las vialidades principales (La Noria (\#19) y Jamaica (\#16)) con representación cercana a la media municipal (hasta 1.18 desviación estándar). En 2010, las localidades alejadas de la frontera con Morelia mantuvieron la mayor representación (Cotzio (\#7), Cuto del Porvenir (\#12), Mesón Nuevo (\#18) y El Puesto (\#30)) hasta a 3.39 desviación estándar; un caso relevante fue el fraccionamiento Las Espigas (\#70) con una representación de 1 desviación estándar, que cambió la tendencia en la distribución de la población desocupada. Este fraccionamiento se creó después del 2005, concentrando una proporción importante de población en edad productiva y vivienda deshabitada ( 2 veces la desviación estándar), evidenciando que la movilidad de los habitantes al periurbano se da por diversos motivos y no solo por la cercanía a los lugares de trabajo. 


\section{Población nacida en otra entidad}

El índice de segregación en los pobladores que nacieron en otra entidad aumentó de 0.69 en el 2000 a 0.76 en 2010. La disimilitud en la distribución de esos habitantes disminuyó del 40\% en el 2000 al $38 \%$ en el 2010. El aislamiento en esta población aumentó de 0.11 a 0.17 , manteniendo mayores posibilidades de contacto entre pobladores del mismo grupo.

Este grupo se ha distribuido con una concentración media, de 0.34 en el 2000 a 0.61 en el 2010, reduciendo la ocupación del centro del municipio. En el 2000 esa población ocupaba localidades tradicionales (Ampliación Jamaica (\#34)) con valores que superaban la media municipal (de hasta 1.24 desviación estándar), resalta el fraccionamiento Campestre Erandeni (\#33) que tenían la mayor concentración de población nacida en otra entidad (de 1 a 4.13 desviación estándar).

En 2010, estos habitantes se concentraban en fraccionamientos a un lado de la carretera (hasta 2.41 desviación estándar), pero la mayor representación era en localidades cercanas a la frontera con Morelia (Fraccionamientos: Erandeni (\#45), Jardín Morelia (\#47), Paseo del Valle (\#59) y Hacienda el Encanto (\#67)). La llegada de los nuevos inmigrantes ha reforzado la antigua localización de los inmigrantes en fraccionamientos cercanos a vías principales y la frontera con Morelia.

\section{Población mayor (de 50 años)}

El índice de segregación también aumentó en los adultos mayores (de 50 años), fue el más alto de los grupos etarios, incrementó de 0.50 en el 2000 a 0.68 en el 2010. La distribución de habitantes mayores de 50 años fue más desigual con respecto al grupo de 15 a 59 años; y más igualitaria con respecto a los menores de 14 años.

Esa distribución ha sido menos concentrada y más centralizada con respecto a los otros grupos etarios; ubicándose principalmente en localidades tradicionales (Tejado de los Izquierdos (\#28), Uruétaro (\#29), El Cuitzillo Grande (\#9) y ExHacienda Guadalupe (\#13)) y en algunos fraccionamientos (Paraíso Escondido (\#24) y Campestre Erandeni (\#33)) con rango de 1 a 2 desviación estándar.

Estos habitantes tienen carencia en servicios de salud y falta de educación básica. Resaltan los casos de localidades tradicionales como Téjaro de los Izquierdos, Uruétaro y El Cuitzillo Grande; y el fraccionamiento Campestre Erandeni, que del 2000 al 2010, han mantenido población de adultos mayores.

\section{Patrones de segregación 1990 al 2010}

Los rasgos de la población en Tarímbaro muestran una desigualdad en la distribución de los habitantes por sus rasgos socioeconómicos, etarios y de migración. De 1990 al 2010, se encontró que los índices de segregación se modificaron, pero mantuvieron valores que van de medios a altos. Ello determinó las diversas dimensiones en que se expresó la segregación, tales como, la concentración, el aislamiento social y la dispersión de los grupos en el territorio.

Para ilustrar como se agrupa la población de acuerdo con los rasgos socioeconómicos, etarios y de migración, se elaboraron grupos por año censal (1990, 2000, 2010) (Figura No 2) que 
tuvieron el mismo comportamiento, estos fueron los patrones de segregación. Se encontró que, en 20 años, los cambios en los atributos personales de la población han reajustado los índices y formas de segregación en el municipio, enfatizando que los asentamientos cercanos a la frontera con Morelia son los espacios más diversos y cambiantes.

Figura No 2.

Patrones de segregación de 1990 al 2010.

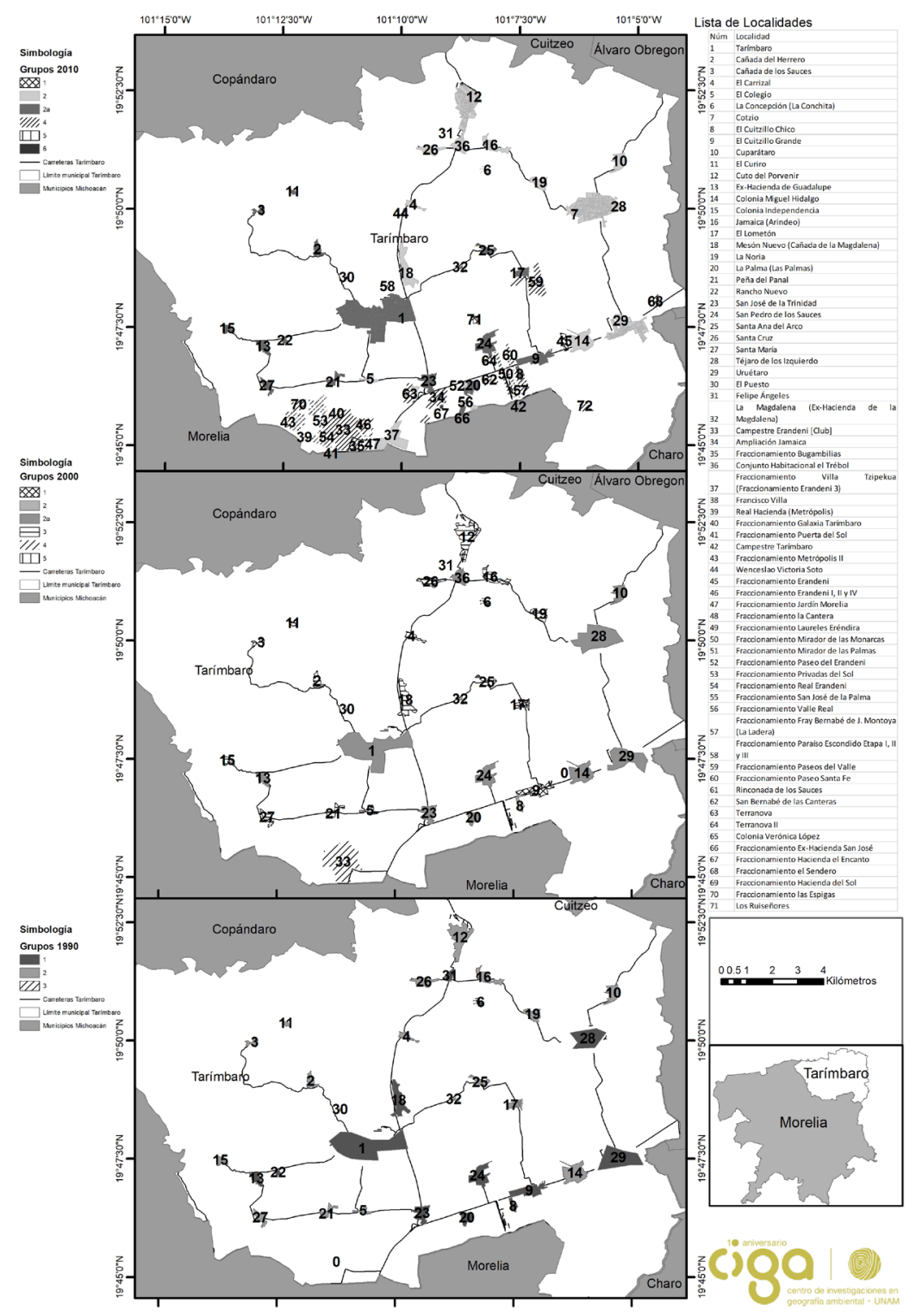

Elaboración propia con base en INEGI (1990, 2000, 2010).

En este sentido, en 1990, el patrón estaba formado por tres grupos: localidades tradicionales mayormente localizadas al centro del municipio y en la frontera con Morelia, con alta representa- 
ción de población con instrucción básica (grupo 1), las características de las viviendas y la población tenían las mejores condiciones a nivel municipal. La población sin instrucción básica tenía alta representación en localidades tradicionales (grupo 2), dispersas en el municipio y alejadas de las vías principales, con las condiciones más precarias en materiales de la vivienda, población sin acceso a servicios de salud e inactiva económicamente. El otro grupo tiene alta representación de población indígena (grupo 3), localizada al Norte del municipio de manera concentrada en una localidad (La Concepción (\#6)); era el más desigual en relación con los materiales de la vivienda, población económicamente activa y sin educación.

De 1990 al 2000, los índices de segregación disminuyeron en la población con educación e indígena, modificando los grupos existentes y reajustando el patrón de segregación. La población indígena se mantuvo concentrada en dos grupos que se diferenciaban por el nivel de instrucción educativa: con educación básica (grupo 1) (El Cuitzillo Grande (\#9)); y habitantes sin educación básica (grupo 5) (La Concepción (\#6)). El primero tenía una localización más cercana a la frontera con Morelia, mientras el grupo 5, estaba alejado del centro de población. Este último tenía las peores condiciones de las viviendas, servicios de salud y su población tenía 50 años y más.

Por otro lado, la población con educación básica estaba representada en dos grupos de localidades tradicionales ( 2 y 2a) cerca de las vías de comunicación y del centro del municipio; se diferenciaban por la representación de población que migró después de 1995 (más alta en el grupo 2a). Estos grupos tenían características de vivienda y población similares a la media municipal; pero el grupo 2a presentaba mayor desigualdad en materiales de la vivienda, servicios de salud y bajos salarios.

La población sin educación (grupo 3), se concentraba en el 50\% de las localidades tradicionales, dispersas y alejadas de las principales vías de comunicación; tenían la mayor representación de viviendas con carencia en servicios y población desocupada del municipio.

Por último, el fraccionamiento Campestre Erandeni (\#33) conformó el grupo de clase alta (grupo 4), localizado en la frontera con Morelia; concentraba la mayor representación de población originaria de otra entidad, altos ingresos (superiores a 6 Salarios Mínimos Mensuales), población en edad productiva (15 a 49 años) y mejores condiciones de vivienda en el municipio.

En la siguiente década, de 2000 al 2010, se incrementó la segregación en población mayor de 60 años, desocupada y nacida en otro estado, modificando nuevamente los grupos existentes y formando otros que expresaban mayor desigualdad.

La población indígena se incrementó a nivel municipal (de 0.18\% en 2000 a 0.32\% en el 2010); la mayor representación se observó en una localidad tradicional (El Puesto \#30) alejada de las principales vías de comunicación (grupo 5); que tenía la mayor representación de habitantes sin educación básica, desocupados y viviendas sin servicios a nivel municipal.

Por otro lado, la población con educación se localizaba en asentamientos tradicionales y en nuevos fraccionamientos (grupo 2 y 2a) localizados de forma dispersa, principalmente en el Centro y Norte del municipio; éstos tenían alta representación de adultos mayores, población desocupada y características de las viviendas similares a la media municipal. 
La población originaria de otra entidad se localizaba en fraccionamientos de clase media y baja (grupo 4) a lo largo de las principales vías de comunicación y la frontera con Morelia; tenía alta representación de población desocupada y en edad productiva (15 a 59 años), así como, viviendas deshabitadas y de uso temporal. Es relevante que la distribución de la población en el 2010 reforzó el acomodo de la población que migró en 1995.

Los fraccionamientos de clase baja creados entre 2005 y 2010 conformaron dos grupos (1, 6): el primero representado por Ex Hacienda San José (\#66) (grupo 6), localizado cercano a la frontera con Morelia, con alta representación de población en edad productiva y con educación básica; mientras que los fraccionamientos Bugambilias (\#35) y Las Espigas (\#70) (grupo 1) tenían representación por debajo de la media municipal en la mayoría de las características socioeconómicas. Ambos grupos presentan altas carencias en servicios de salud y en la vivienda, además se distinguen por tener la mayor representación de vivienda deshabitada en el municipio.

En el periodo analizado, se observó que la modificación en los índices de segregación fueron determinantes para que la población indígena, los adultos mayores y la población desocupada que acumulan desventajas por su ubicación espacial, disminuyeran las posibilidades de contactos con otro grupo e incrementaran la falta de acceso a servicios de salud y en la vivienda.

La estructura espacial de las ciudades ha cambiado por ajustes económicos, políticos y la conectividad global (Sabatini, 2006; Bähr y Borsdorf, 2005; Janoschka, 2002), generando en América Latina, dos transiciones: la primera, urbana por la migración rural-urbana; y la segunda, demográfica, por las tasas de crecimiento y envejecimiento poblacional (ONU y CEPAL, 2016), ambas imbricadas, generadas por procesos externos e internos.

Las ciudades de tamaño mediano no son la excepción, en su territorio periurbano las modificaciones han sido más abruptas, porque mantienen características rurales en los paisajes, en las condiciones de la vivienda y en las carencias de servicios. Además, el espacio es ocupado por diversos grupos de habitantes (tradicionales y recién llegados) que se diferencian por sus atributos personales. Acorde con otras investigaciones en ciudades de tamaño mediano mexicanas (Domínguez, 2017; Culiacán, Pérez-Tamayo, Gil-Alonso y Bayona-I-Carrasco, 2017) estos cambios se materializan en los patrones de segregación, donde la condición migratoria y el origen étnico de la población son atributos importantes para la diferenciación social.

En el caso de Tarímbaro, con un crecimiento por arriba de la media metropolitana, mantiene las estructuras tradicionales y paisajes rurales. La población en ese municipio experimenta modificaciones en sus atributos personales por las dinámicas de las localidades tradicionales, tales como el envejecimiento, la movilidad de población indígena y la mejora en niveles educativos; pero también, producto de la expansión urbana: como la inmigración de pobladores de otros municipios del país, y la llegada de población económicamente activa, joven y adulta, en los nuevos fraccionamientos (clase media- baja y de altos ingresos). Estos cambios alteran los índices, reforzando antiguas y nuevas formas de segregación.

Las modificaciones de la estructura urbana promueven que habitantes de diferentes grupos estén más próximos, lo que disminuye la desigualdad en la distribución, y por lo tanto, se encuentren menos segregados aunque se incremente la diferenciación social entre los grupos 
(Sabatini y Brain, 2008). Sin embargo, la disminución de la segregación no ocurre en todos los rasgos sociales.

En resumen, de 1990 al 2010, la disminución de la segregación en Tarímbaro se expresó en dos direcciones, por un lado, en las características de la población que incrementan la diferenciación en su distribución (población desocupada, nacida en otra entidad y mayor de 50 años); y por el otro lado, en los atributos de la población que disminuyen la desigualdad en la distribución espacial (población indígena, con escolaridad y económicamente activa).

Estos atributos fueron determinantes para formar los patrones socio-espaciales. Primero, al Centro y Norte, la igualdad en localidades tradicionales y fraccionamientos, que representan el $50 \%$ de los asentamientos municipales, mejoraron sus niveles de educación y características de las viviendas. Segundo, alta concentración de población indígena en un asentamiento sin educación y carencias en servicios. Por último, concentración de población en nuevos fraccionamientos, cercanos a la frontera con Morelia, de clase baja y media, población con educación básica y en edad productiva, pero con alta representación de vivienda deshabitada y carencias en servicios. Estos patrones son resultado de movimientos reiterativos y han reforzado la ubicación que ha tenido la población migrante e indígena desde 1990 en el municipio.

Particularmente, las dimensiones analizadas mostraron las diversas expresiones de la segregación: en los habitantes indígenas distribuidos de forma concentrada y de la población inmigrante localizada con alta concentración en la frontera con Morelia.

Hallazgos que coinciden con otras investigaciones internacionales en ciudades de tamaño mediano, que observan como la concentración de grupos étnicos e inmigrantes repercute en las condiciones de la vivienda (Bolt y Kempen, 1997); así como, con estudios de ciudades de tamaño mediano mexicanas (Domínguez, 2017) que observan a los indígenas distribuidos de manera polinuclear en antiguas localidades y los inmigrantes localizados reiterativamente en las mismas zonas.

Respecto, a la distribución de los adultos mayores, ésta es concentrada en antiguas localidades, de manera segregada espacialmente, contraponiendo otros estudios que mencionan que la representación de esta población decrece en las zonas alejadas del centro de Morelia (Álvarez de la Torre, 2017); y evidenciando como esa localización contribuye a limitar y hacer poco sostenidos los contactos sociales en esa población (Pola- Villaseñor et al., 2017).

\section{Conclusiones}

El objetivo propuesto en este trabajo fue analizar los cambios en el patrón de segregación en un municipio periurbano de la ZMMor, a partir de la disimilitud en los atributos personales y sus implicaciones en el acceso a servicios y condiciones de las viviendas, que se cumple con los siguientes hallazgos. Primero, se encontró, que la heterogeneidad de atributos personales de la población, de un municipio en el periurbano de una ciudad media, determina la segregación en esos territorios. Considerar a los atributos personales como elementos centrales de análisis, permitió superar las limitaciones de otros estudios que solo consideran al ingreso, la clase social 
o las condiciones de la vivienda, para analizar la segregación (Rubalcaba y Schteingart, 2012; Aguilar, Romero y Hernández, 2015), porque mostró la relevancia de procesos como la migración, el envejecimiento y la pertenencia a un grupo indígena, en el periurbano.

No obstante, esa heterogeneidad provoca dos procesos: el incremento en la disímil distribución poblacional causado por atributos como la desocupación económica, la migración y la población adulta (más 50 años); por otro lado, una igualdad en la distribución poblacional, producto de atributos como hablar una lengua indígena, tener escolaridad básica y ocupación económica.

En el segundo hallazgo, se encontró que los cambios en la heterogeneidad de la población son guías para los patrones de segregación. Es decir, se observó que dichos patrones se modifican en tres direcciones: igualdad de localidades tradicionales y nuevos fraccionamientos que aglomeran población adulta (más 50 años), productiva y con educación básica que mejoran sus condiciones de la vivienda, ellos respetan el $98 \%$ de la población municipal; por otro lado, una alta concentración de población indígena en localidades alejadas del centro, que ven mermadas sus condiciones de vida y que representa menos del $1 \%$ de la población; y por último, la creación de nuevos fraccionamientos en la frontera con Morelia, la zona más desigual, habitados por jóvenes, recién llegados, que enfrentan carencias en su vida diaria y que no rebasan el 1\% de la población.

Lo anterior es el resultado de procesos construidos históricamente, por ejemplo, la población indígena y sin escolaridad ha enfrentado carencias en sus condiciones de vida; ello permite reconocer que el proceso de periurbanización está reforzando antiguas desiguales municipales; al mismo tiempo, que incorpora otras nuevas, con la población migrante que llega a los fraccionamientos urbanos en la frontera con Morelia.

Estos resultados son útiles porque sirven para relacionar los patrones de segregación con otros aspectos, como: la gestión de la urbanización en esos territorios; el costo que los gobiernos locales deben cubrir para dotar de infraestructura y servicios básicos y empleo a la población dispersa (CEPAL, 2016); y la distribución de las demandas del mercado de vivienda (Bolt y Kempen, 1997) en la frontera con Morelia. Por último, el análisis de los patrones de segregación a escala municipal es un preámbulo para entender procesos sociales y culturales que ocurren a escalas menores como el barrio y la familia.

\section{Bibliografía}

AGUILAR, I. El proceso de urbanización del Área Metropolitana de Monterrey: Algunas reflexiones de la experiencia reciente. En: AGUILAR A. Procesos metropolitanos y grandes ciudades. Dinámicas recientes en México y otros países. México: UNAM y MA. Porrúa, 2004, p. 219-264.

AGUILAR, A. \& MATEO, P. Diferenciación sociodemográfica del espacio urbano de la Ciudad de México, Eure, 2011, N¹10, p. 5-30.

AGUILAR, A. \& LÓPEZ, F. The city-region of Mexico City: social inequality and vacuum in development planning. International Development Planning Review, 2018, Vol. 40, p. 52-74. 
AGUILAR, A., ROMERO, P. \& HERNÁNDEZ, J. Segregación socio-residencial en la Ciudad de México. Dinámica del patrón territorial a nivel local 2000-2010. En: AGUILAR A. y ESCAMILLA, I. Segregación Urbana y Espacios de Exclusión. Ejemplos de México y América Latina. México: Instituto de Geografía- UNAM y MA Porrúa, 2015, p.73-102.

ÁLVAREZ DE LA TORRE, G. Morfología y estructura urbana en las ciudades medias mexicanas. Región y Sociedad, 2017, Vol. 29, p. 153-191.

BADAWY, A., ABDEL-SALAM, H. \& AYAD, H. Investigation the impact of urban planning policies on urban division in Cairo, Egypt: The case of El-Maadi and Dar El-Salam, Alexandria Engineering Journal, 2015, No 54. p. 1225-1232.

BÄHR, J. \& BORSDORF, A. La Ciudad Latinoamericana, la construcción de un modelo, vigencia y perspectivas. Urbes. 2005, No 2. p. 207-221.

BOLT, G. \& KEMPEN, R. Segregation and Turk's housing condition in middle -sized Dutch cities, Journal of Ethnic and Migration Studies, 1997, Co. 23, p. 363-384.

CAVAILHÉS, J., PEETERS, D., SÉKERIS, E. \& THISSE, J. The periurban city: why to live between the suburbs and the countryside, Regional Science and Urban Economics, 2004, p. 681-703.

CONSEJO NACIONAL DE EVALUACIÓN DE LA POLÍTICA DE DESARROLLO SOCIAL (CONEVAL). Canasta alimentarias y no alimentarias, observadas y normativas. México: CONEVAL, 2017.

DA GAMA, H. Environmental Implications of Peri-urban sprawl and the Urbanization of Secondary Cities in Latin America, Inter-American Development Bank (IDB), 2011.

DOMÍNGUEZ, M. Las dimensiones espaciales de segregación residencial en la ciudad de Mérida, Yucatán, a principios del siglo XXI. Península, 2017, No. 12, p. 147-188.

GARCÍA, L. \& RODRÍGUEZ, L. Segregación y entropía en Bogotá. En: RUBIANO, M (compiladora), Segregación socioespacial. Miradas cruzadas desde Rio de Janeiro, Bogotá y sus áreas de influencia metropolitana, Bogotá: Universidad Piloto de Colombia, 2017, p. 215 -242.

HERNÁNDEZ, J. MARTínEZ, B., MÉNDEZ, J., PÉREZ, R., RAMÍREZ, J. \& NAVARRO, H.. Rurales y periurbanos: una aproximación al proceso de conformación de la periferia poblana. Papeles de población, 2009, No. 61, p. 275-295.

HILL, E. \& BRENNAN, J. America's Central Cities and Location of Work: Can Cities Compete with Their Suburbs?, Journal of the American Planning Association, 2005, No 71, p. 411-432.

INSTITUTO NACIONAL DE ESTADÍSTICA Y GEOGRAFÍA (INEGI). Censo de Población y Vivienda, 1990. Disponible en Internet: http://www.beta.inegi.org.mx/proyectos/ccpv/1990/.

INSTITUTO NACIONAL DE ESTAdíSTICA Y GEOGRAFÍA (INEGI). Censo de Población y Vivienda, 2000. Disponible en Internet: http://www.beta.inegi.org.mx/proyectos/ccpv/2000/default.html. 
INSTITUTO NACIONAL DE ESTAdístICA Y GEOGRAFía (INEGI). Censo de Población y Vivienda, 2010. Disponible en Internet: http://www.beta.inegi.org.mx/proyectos/ccpv/2010/default.html.

INSTITUTO NACIONAL DE ESTADÍSTICA Y GEOGRAFÍA (INEGI). Glosario. 2018. Disponible en Internet: http://www.inegi.org.mx/est/contenidos/espanol/sistemas/cem07/texcom/glosario/glosario. htm.

JANOSCHKA, M. El nuevo modelo de la ciudad latinoamericana: fragmentación y privatización. Eure. 2002 No 85. Disponible en Internet: http://www.scielo.cl/scielo.php?script=sci_arttext\&pi$d=S 0250-71612002008500002$.

LICHTER, D. \& BROWN, D. Rural American in an Urban Society: Changing Spatial and Social Boundaries, Annual Review of Sociology, 2011, Vol. 37, p. 565-592.

MADRAZO, B. \& KEMPEN, R. Explaining divided cities in China, Geoforum, 2011, p. 158-168.

MARTÍNEZ, S. \& MONROY-ORTIZ, R. La expansión urbana sobre el campo. La otra cara de la crisis agrícola, Estudios agrarios, 2009, p. 29-46.

MASSEY, D. \& DENTON, N. The Dimensions of Residential Segregation, Social Forces, 1988, No 2, p. 281-315.

MASSEY, D., ROTHWELL, J. \& DOMINA, T. The Changing of the Segregation in the United States, The Annals of the American Academy, 2009, p. 74-90.

NELLES, J. Cooperation and Capacity? Exploring the Sources and Limits of City-Region Governance Partnerships, International Journal of Urban and Regional Research, 2012, Vol. 37, p. 1349-1367.

NELLO, O. Los confines de la ciudad sin confines. Estructura urbana y límites administrativos en la ciudad difusa, En: MONCLÚS, F. La ciudad dispersa, Barcelona: Centro de Cultura Contemporánea de Barcelona, 1998, p. 35-57.

ORGANIZACIÓN DE NACIONES UNIDAS (ONU) y COMISIÓN ECONÓMICA PARA AMÉRICA LATINA Y EL CARIBE (CEPAL). Ciudades sostenibles con igualdad en América Latina y el Caribe. 2016.

PARR, J. Perspectives on the city-region, Regional Studies, 2005, Vol. 39, p. 555-566.

POLA-VILLASEÑOR, S., MÉNDEZ-LEMUS, Y. \& VIEYRA, A. Acceso al suelo ejidal periurbano: análisis desde el capital social, Economía, Sociedad y Territorio, 2017, No. 54, p. 429-460.

ROBERTS, B. Gestionando Sistemas de Ciudades Secundarias. Washington DC: Cities Alliance/ Banco Interamericano de Desarrollo, 2015.

RUBALCABA, R. \& SCHTEINGART, M. Ciudades divididas: Desigualdad y segregación social en México. México: Colegio de México, 2012. 
SABATINI, F. La segregación social del espacio en las ciudades de América Latina. Banco Interamericano de Desarrollo (BID). BID.2006.

SABATINI, F. \& BRAIN, I. La segregación, los guetos y la integración social urbana: mitos y claves, Eure, 2008, No. 103, p. 5-26.

SABATINI, F. La ruptura del patrón de segregación y su significado teórico y práctico. En: AGUILAR A. y ESCAMILLA, I. Segregación Urbana y Espacios de Exclusión. Ejemplos de México y América Latina. México: Instituto de Geografía- UNAM y MA Porrúa, 2015, p.25-46.

SAVAGE, M., DEVINE, F., CUNNINGHAM, N., TAYLOR, M., LI, Y., HJELLBREKKE, J., LE ROUX, B., FRIEDMAN, S. \& MILES, A. A new model of social class? Finding from the BBC's Great British Class Survey experiment. Sociology, 2013, No 47, p. 219-250.

SCHTEINGART, M. La división social del espacio en ciudades mexicanas: un balance explicativo desde una perspectiva latinoamericana, En: AGUILAR A. y ESCAMILLA, I. Segregación Urbana y Espacios de Exclusión. Ejemplos de México y América Latina. México: Instituto de Geografía- UNAM y MA Porrúa, 2015, p.47-72

SECRETARÍA DE DESARROLLO SOCIAL (SEDESOL) y ONU-HABITAT. Estado de las ciudades de México. México: SEDESOL, ONU-HABITAT, 2011.

SECRETARÍA DE GOBERNACIÓN (SEGOB), SECRETARÍA DE DESARROLLO AGRARIO, TERRITORIAL Y URBANO (SEDATU) y CONSEJO NACIONAL DE POBLACIÓN (CONAPO). Sistema Urbano Nacional. México: SEGOB, SEDATU y CONAPO, 2018.

SMITH, D. Urban Environments: Issues on the Peri-Urban Fringe, Annual Review of Environment and Resources, 2008, Vol. 33, p. 167-185.

UNITED NATIONS (UN). Population Division, World Urbanization Prospects; The 2014 Revision, Highlights, Department of Economic and Social Affairs, 2014.

UNITED CIUDADES AND LOCAL GOVERNMENTS (UCLG), CITÉ ET GOUVERNEMENTS LOCAUX UNIS (CGLU). Co-Creando el Futuro Urbano. La Agenda de las Metrópolis, las Ciudades Intermedias y los Territorios. Barcelona: UCLG, CGLU, 2016.

VARGAS, G. El proyecto de ciudad a la ciudad sin proyecto: El desarrollo histórico territorial de la traza urbana de la ciudad de Valladolid-Morelia 1541-2009. A. VIEYRA Y A. LARRAZÁBAL (coord.) Urbanización, sociedad y ambiente. Experiencias en ciudades medias, México, CIGA, INECC, 2014, p. $47-85$.

VERGARA-ERICES, L. \& GARÍN, A. Vivienda social y segregación socioespacial en una ciudad pequeña: el caso de Angol, Chile. Polis, 2016, No 44, p. 1-25. 
WU, Q., CHENG, J., CHEN, G., HAMMEL, D. \& WU, X. Socio-spatial differentiation and residential segregation in the Chinese city based on the 2000 community-level census data: A case study of the inner city of Nanjing. Cities, 2014, No 39, p. 109-119.

\section{Agradecimiento}

Los autores agradecen el financiamiento del Programa PAPIIT-DGAPA-UNAM al proyecto de investigación: "La segregación en el periurbano de las ciudades medias mexicanas, los casos de Morelia y Oaxaca" (IA301419), del cual forma parte este artículo. 
